\title{
Why is it Crucial to Use Personalized Occlusion Pressures in Blood Flow Restriction (BFR) Rehabilitation?
}

\author{
James A. McEwen ${ }^{1,3}$ • Johnny G. Owens ${ }^{2}$ Jeswin Jeyasurya ${ }^{3}$ \\ Received: 25 August 2017 / Accepted: 28 March 2018 / Published online: 9 April 2018 \\ (c) The Author(s) 2018
}

\begin{abstract}
An increasing amount of evidence has been shown to support the use of blood flow restriction (BFR) in combination with low-load resistance exercise to enhance morphological and strength responses. The BFR technique involves applying a tourniquet cuff to a limb and pressurizing it with a tourniquet instrument to restrict, but not fully occlude, arterial blood flow into the limb during rehabilitative exercise. A review of BFR rehabilitation literature shows that inconsistencies exist in methodology, equipment and in levels of restriction pressure used. Current non-personalized methodologies of setting BFR pressure may occlude rather than restrict blood flow, increasing the risk of injury during rehabilitation. Furthermore, these non-personalized methods of setting pressure do not provide a consistent stimulus within and across patients, reducing the efficacy of the BFR rehabilitation and inhibiting the meaningful comparison of a full range of BFR studies. A restriction pressure level set for each individual patient, based on a percentage of limb occlusion pressure (LOP) measured at rest, and applied using a surgical-grade tourniquet cuff, enables those individual patients to receive a safe and consistent BFR stimulus compared to other methods of setting the restriction pressure level. In view of the above, it is crucial to use surgical-grade tourniquet technology with automatic LOP measurement capability, adapted to incorporate and deliver optimal protocols, for safe and effective application of BFR to consistently achieve optimal patient outcomes in rehabilitation.
\end{abstract}

Keywords Tourniquet $\cdot$ Personalized $\cdot$ Rehabilitation $\cdot$ Blood flow restriction (BFR) $\cdot$ Recovery $\cdot$ Muscle strength

\section{Introduction}

Periods of reduced activity are common following surgery or injury. Physical inactivity leads to muscle atrophy, and inactivity caused by an unloading of body weight is associated with numerous health consequences including changes in the quality and quantity of muscle and bone and a reduced ability for rehabilitative exercise [1].

Resistance rehabilitation is used to recover muscle size and strength following injury or surgery. Typically, a person is required to lift loads at or above $65 \%$ of their one

Jeswin Jeyasurya

jeswin.jeyasurya@wclinical.com

1 Department of Orthopaedics and Electrical and Computer Engineering, University of British Columbia, 2775 Laurel Street, Vancouver, BC V5Z 1M9, Canada

2 Owens Recovery Science, 321 6th Street, San Antonio, TX 78215, USA

3 Western Clinical Engineering, 207-1099 West 8th Avenue, Vancouver, BC V6H 1C3, Canada repetition maximum (1RM) to have noticeable increases in muscle size and strength [2]. However, during rehabilitation from injury patients may be limited to performing low-load resistance rehabilitative exercises in which strength and size benefits are less evident compared with high-load resistance rehabilitative exercise.

An increasing amount of evidence has been shown to support the use of blood flow restriction (BFR) in combination with low-load resistance rehabilitation $(\sim 20-40 \%$ 1RM $)$ to augment morphological and strength responses [3, 4]. Studies have also shown that applying BFR without rehabilitative exercise after lower limb surgery [5] or after limb immobilization $[6,7]$ effectively diminishes muscle atrophy due to disuse and associated loss of muscle strength. This evidence indicates that BFR may also be useful for rehabilitation without exercise.

The BFR technique involves applying a tourniquet cuff to a limb and pressurizing it with a tourniquet instrument to restrict, but not fully occlude, arterial blood flow into the limb during rehabilitative exercise. Physiologically, it is hypothesized that the ischemic and hypoxic muscular 
environment created during BFR causes high levels of metabolic stress and mechanical tension when used in tandem with exercise. Metabolic stress and mechanical tension have both been described as 'primary hypertrophy factors' that are theorized to activate other mechanisms that induce muscle growth. However, presently these associations are primarily hypothetical and specific identification of the mechanisms is currently lacking [4]. Nonetheless, these findings have significant implications in that low-load rehabilitative exercise with BFR can facilitate muscular changes in populations where high mechanical loads may be contraindicated or not possible, including post-operative rehabilitation patients and the elderly [3].

Although clinical interest in the use of BFR exercise as a rehabilitation tool has greatly increased in recent years, a review of BFR rehabilitation literature shows that inconsistencies exist in methodology, equipment and in levels of restriction pressure used. For example Jessee et al. [8] summarized fifteen recently published BFR studies in the upper body and cuff pressures ranged widely. Some studies used a pressure applied with a tourniquet cuff at a level set as a percentage of personalized limb occlusion pressure (LOP), other studies used a fixed cuff pressure applied with cuffs having a variety of sizes and shapes, and a few studies set pressure based on systolic blood pressure using old formulas that have been proven inaccurate, unreliable and largely discontinued in surgical tourniquet settings [9-11]. These inconsistencies in methodology and equipment have made it difficult to apply a safe and consistent BFR stimulus to patients, they prevent a controlled comparison of different BFR protocols, and thus they limit the identification and delivery of optimal patient outcomes.

This paper explains why it is crucial to use surgical-grade tourniquet technology with automatic LOP measurement capability, adapted to incorporate and deliver optimal protocols, for safe and effective application of BFR to consistently achieve optimal patient outcomes in rehabilitation.

\section{Limb Occlusion Pressure (LOP)}

To overcome the above described inconsistencies, many studies $[8-10,12]$ have recommended the use of personalized pressures based on LOP for BFR rehabilitation. LOP is defined as the minimum pressure required, at a specific time in a specific tourniquet cuff applied to a specific patient's limb at a specific location, to stop the flow of arterial blood into the limb distal to the cuff. LOP is affected by variables including the patient's limb characteristics; characteristics of the selected tourniquet cuff, including shape, width, length, presence or absence of circumferential bladder and internal stiffener; the technique of application of the cuff to the limb; physiologic characteristics of the patient including blood pressure and limb temperature; and other clinical factors (for example, the extent of any elevation of the limb during LOP measurement and the extent of any limb movement during measurement) [11].

\section{The Need for Personalized Pressures}

A restriction pressure level set for each individual patient, based on a percentage of LOP measured at rest, and applied using a surgical-grade tourniquet cuff, enables those individual patients to receive a safe and consistent BFR stimulus compared to other methods of setting the restriction pressure level [10]. Current non-personalized methods of setting BFR pressures have significant safety and efficacy issues.

\subsection{Safety-Related Aspects of Existing BFR Techniques}

The primary safety issue with current methodologies of setting restriction pressures for BFR is the potential of using pressures that are higher than LOP. An analysis of previous studies using an arbitrary fixed pressure of $200 \mathrm{mmHg}$ or using a percentage of brachial systolic blood pressure (SBP) (e.g. 130\% of SBP) and cuffs of differing widths showed that these methodologies of setting pressure may result in a significant number of subjects having cuff pressures above the LOP, thus occluding rather than restricting blood flow during the rest period and possibly during exercise itself [13]. Other non-pneumatic bands and elastic wraps [10] apply unknown pressures to the limb which have been shown in some instances to be hazardously higher than the LOP [11, $14,15]$.

It is well established in the literature that higher levels of tourniquet pressure and higher pressure gradients underneath tourniquet cuffs are associated with a higher risk of nerverelated injury [11]. Although injury from BFR rehabilitation is uncommon, use of pressures that are unnecessarily high increases the risk of detrimental side effects including possible nerve injury and ischemic injury [11].

Furthermore, the use of pressures that occlude rather than restrict blood flow is associated with other limitations and hazards. Complete arterial occlusion reduces the effectiveness of the BFR intervention and can cause the formation of a thrombus. Also, unnecessarily high levels of limb compression may cause a slowing of nerve conduction velocity, potentially detrimental for long duration BFR rehabilitation. Further, higher pressures place a greater demand on the cardiovascular system compared to lower pressures during BFR rehabilitation [16]. 


\subsection{Efficacy-Related Aspects of Existing BFR Techniques}

Loenneke et al. $[9,10]$ demonstrated that setting BFR pressure as a function of blood pressure or at a fixed pressure does not provide a consistent stimulus across patients because these methods of setting pressure neglect important factors that affect LOP, including limb circumference and cuff width, Fig. 1. This confirms what has been well established in the surgical tourniquet literature on LOP [11]. Fatela et al. [12] analyzed the effect of relative BFR pressure on the acute neuromuscular response to BFR resistance exercise and showed that muscular activation and neuromuscular fatigue varies as a function of relative BFR. Consequently, Fatela et al. [12] concluded that it is crucial to determine individual levels of vascular restriction, by quantifying the resting LOP, before engaging in BFR rehabilitative exercise.

\section{Benefits of Personalized Pressures}

There are three primary benefits of using personalized pressures based on a relative percentage of LOP, determined automatically on a resting patient by a surgicalgrade tourniquet instrument, and applied safely and consistently by a surgical-grade tourniquet cuff, Fig. 2. First, the use of such tourniquet instruments and cuffs are based on decades of experience in surgical settings, and assures the safe, accurate, and reliable application of pressure to a patient's limb [11]. Setting and regulating the pressure as a predetermined percentage of the LOP can help avoid adverse events that may result from inadvertently applying pressures that result in complete arterial occlusion [8]. Second, the application of a consistent level of restriction pressure limits variability in BFR intensity for individual patients, since muscular activation, as well as neuromuscular fatigue, varies as a function of relative BFR intensity [12]. Third, accurately applying a consistent level of restriction pressure enables the outcomes and results of a full range of BFR studies to be compared on a meaningful basis so that optimal protocols can be identified and applied [17].

\section{Limitations of Other Approaches}

In early studies the most commonly used method for determining LOP to set restriction pressure was based on the use of Doppler ultrasound by a specifically trained clinician. However, this method is time-consuming, requires additional equipment, and the accuracy of LOP measurement is highly dependent on the specific training and experience of the measuring clinician. Alternatively, Jessee et al. [8] developed equations to predict a patient's LOP, taking into account some of the determinants of LOP investigated in their study, but these equations do not account for all variables known to affect LOP [11] and their application may be too complex and time-consuming for routine clinical use. Additionally, some low-cost, non-tourniquet cuffs [18] and other devices such as elastic knee wraps [19] have been proposed for BFR rehabilitation, but their effectiveness is unproven and they present safety hazards, Fig. 3. This is because they do not have the ability to automatically take into account each patient's LOP when setting the restriction
Fig. 1 Limb occlusion pressure (LOP) versus the ratio of tourniquet cuff width to limb circumference. For any given limb circumference, the tourniquet pressure required to stop arterial blood flow decreases as the width of the tourniquet cuff increases. Reproduced with permission from Graham et al. [20]

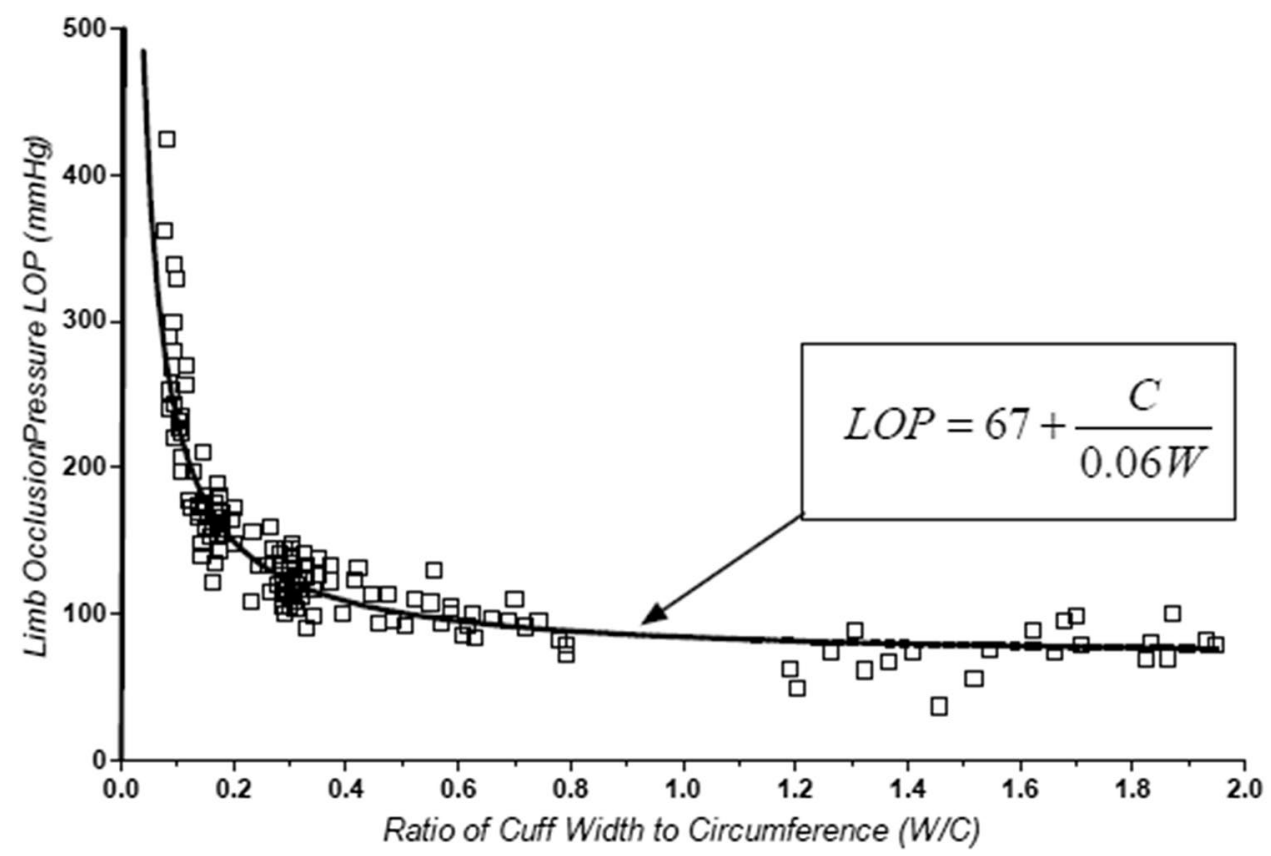


Fig. 2 Modern surgical-grade tourniquet instrument and cuff adapted for BFR rehabilitation, showing elements that provide improved safety, accuracy and reliability to consistently achieve optimal patient outcomes
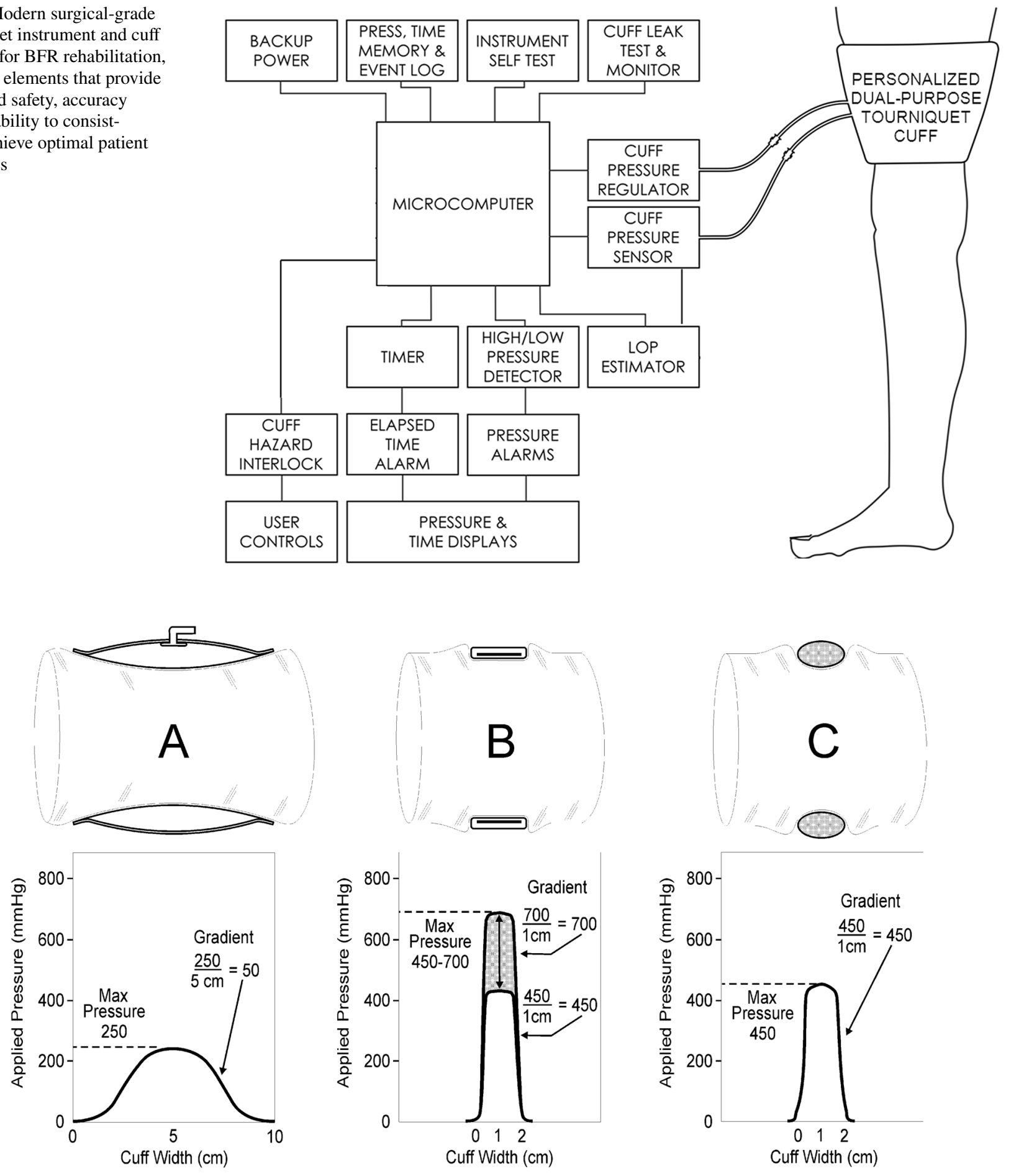

Fig. 3 A comparison of applied pressures and pressure gradients typically produced by $\mathbf{a}$ a modern pneumatic surgical tourniquet cuff, $\mathbf{b}$ a non-pneumatic, non-surgical strap-type tourniquet and $\mathbf{c}$ a non-pneumatic elastic ring designed to combine exsanguination and tourniquet functions. Each tourniquet was selected and applied as recommended

pressure level for individual patients, they apply unknown and inconsistent pressures to a patient's limb that can be much higher or much lower than the intended restriction by the respective manufacturer to stop arterial blood flow in an upper limb. Higher levels of pressure and higher pressure gradients are associated with higher probabilities of patient injuries. Reproduced with permission from McEwen and Casey [15]. CMBEC32, Calgary, Canada, 2009 May 20-22

pressure [11, 14], and they lack important safety features proven in surgical-grade tourniquet instruments and cuffs such as safe limits on pressures and protocols, accurate 
pressure regulation and low pressure levels and gradients beneath cuffs [11].

\section{Conclusion}

In view of the above, it is crucial to use surgical-grade tourniquet technology with automatic LOP measurement capability, adapted to incorporate and deliver optimal protocols, for safe and effective application of BFR to consistently achieve optimal patient outcomes in rehabilitation.

\section{Compliance with Ethical Standards}

Conflict of interest Author Johnny Owens is a shareholder of Owens Recovery Science and is a medical consultant for Delfi Medical Innovations Inc. Owens Recovery Sciences has a financial relationship with Delfi Medical Innovations Inc. Author Jeswin Jeyasurya is an employee of Western Clinical Engineering Ltd.; In addition, Mr. Jeyasurya has a patent US 9,039,730 issued and a patent application PCTCA/2015/050458 filed. Author James McEwen is the president and a shareholder of Western Clinical Engineering Ltd. In addition, Dr. McEwen has a patent US 9,039,730, and a patent PCT $\mathrm{CA} / 2015 / 050458$ pending and serves as a board member and shareholder of Delfi Medical Innovations Inc. which has a financial relationship with Owens Recovery Sciences.

Open Access This article is distributed under the terms of the Creative Commons Attribution 4.0 International License (http://creativeco mmons.org/licenses/by/4.0/), which permits unrestricted use, distribution, and reproduction in any medium, provided you give appropriate credit to the original author(s) and the source, provide a link to the Creative Commons license, and indicate if changes were made.

\section{References}

1. Loenneke, J., Abe, T., Wilson, J., Thiebaud, R., Fahs, C., Rossow, L., et al. (2012). Blood flow restriction: An evidence based progressive model. Acta Physiologica Hungarica, 99(3), 235-250.

2. ACSM. (2009). American College of Sports Medicine position stand. Progression models in resistance training for healthy adults. Medicine and Science in Sports and Exercise, 41, 687-708.

3. Scott, B. R., Loenneke, J. P., Slattery, K. M., \& Dascombe, B. J. (2015). Exercise with blood flow restriction: An updated evidence-based approach for enhanced muscular development. Sports Medicine, 45(3), 313-325.

4. Hughes, L., Paton, B., Rosenblatt, B., Gissane, C., \& Patterson, S. D. (2017). Blood flow restriction training in clinical musculoskeletal rehabilitation: A systematic review and meta- analysis. British Journal of Sports Medicine. https://doi.org/10.1136/bjspo rts-2016-097071.

5. Takarada, Y., Takazawa, H., \& Ishii, N. (2000). Applications of vascular occlusion diminish disuse atrophy of knee extensor muscles. Medicine and Science in Sports and Exercise, 32(12), 2035-2039.
6. Kubota, A., Sakuraba, K., Koh, S., Ogura, Y., \& Tamura, Y. (2011). Blood flow restriction by low compressive force prevents disuse muscular weakness. Journal of Science and Medicine in Sport, 14(2), 95-99.

7. Kubota, A., Sakuraba, K., Sawaki, K., Sumide, T., \& Tamura, Y. (2008). Prevention of disuse muscular weakness by restriction of blood flow. Medicine and Science in Sports and Exercise, 40(3), 529-534.

8. Jessee, M. B., Buckner, S. L., Dankel, S. J., Counts, B. R., Abe, T., \& Loenneke, J. P. (2016). The influence of cuff width, sex, and race on arterial occlusion: Implications for blood flow restriction research. Sports Medicine, 46(6), 913-921.

9. Loenneke, J. P., Wilson, J. M., Wilson, G. J., Pujol, T. J., \& Bemben, M. G. (2011). Potential safety issues with blood flow restriction training. Scandinavian Journal of Medicine and Science in Sports, 21(4), 510-518.

10. Loenneke, J. P., Fahs, C. A., Rossow, L. M., Sherk, V. D., Thiebaud, R. S., Abe, T., et al. (2012). Effects of cuff width on arterial occlusion: Implications for blood flow restricted exercise. European Journal of Applied Physiology, 112(8), 2903-2912.

11. Noordin, S., McEwen, J. A., Kragh, J. F., Eisen, A., \& Masri, B. A. (2009). Surgical tourniquets in orthopaedics. Journal of Bone and Joint Surgery. American Volume, 91(12), 2958-2967.

12. Fatela, P., Reis, J. F., Mendonca, G. V., Avela, J., \& Mil-Homens, P. (2016). Acute effects of exercise under different levels of bloodflow restriction on muscle activation and fatigue. European Journal of Applied Physiology, 116(5), 985-995.

13. Loenneke, J. P., Fahs, C. A., Rossow, L. M., Thiebaud, R. S., Mattocks, K. T., Abe, T., et al. (2013). Blood flow restriction pressure recommendations: A tale of two cuffs. Frontiers in Physiology, 4, 249. https://doi.org/10.3389/fphys.2013.00249.

14. McEwen, J. A. (1981). Complications of and improvements in pneumatic tourniquets used in surgery. Medical Instrumentation, 15(4), 253-257.

15. McEwen, J., \& Casey, V. (2009). Measurement of hazardous pressure levels and gradients produced on human limbs by non-pneumatic tourniquets. In Proceedings of the 32nd Conference of the Canadian Medical and Biological Engineering Society Calgary, Canada, May 20-22, 2009 (pp. 1-4).

16. Loenneke, J. P., Thiebaud, R. S., Abe, T., \& Bemben, M. G. (2014). Blood flow restriction pressure recommendations: The hormesis hypothesis. Medical Hypotheses, 82(5), 623-626.

17. McEwen, J., Owens, J., \& Jeyasurya, J. (2016). How can personalized tourniquets accelerate the rehabilitation of wounded warriors, professional athletes, and orthopaedic patients. In CMBEC 2016, Calgary, AB, May 24-27 (pp. 1-4).

18. Loenneke, J. P., \& Pujol, T. J. (2009). The use of occlusion training to produce muscle hypertrophy. Strength \& Conditioning Journal, 31(3), 77-84.

19. Takano, H., Morita, T., Iida, H., Asada, K. I., Kato, M., Uno, K., et al. (2005). Hemodynamic and hormonal responses to a shortterm low-intensity resistance exercise with the reduction of muscle blood flow. European Journal of Applied Physiology, 95(1), 65-73.

20. Graham, B., Breault, M. J., McEwen, J. A., \& McGraw, R. W. (1993). Occlusion of arterial flow in the extremities at subsystolic pressures through the use of wide tourniquet cuffs. Clinical Orthopaedics and Related Research, 286, 257-261. 